\title{
PREDICTING SPECTRAL AND PROSODIC PARAMETERS FOR UNIT SELECTION IN SPEECH SYNTHESIS
}

\author{
Minghui Dong and Haizhou Li \\ Institute for Infocomm Research $\left(\mathrm{I}^{2} \mathrm{R}\right), \mathrm{A}^{*} \mathrm{STAR}, 1$ Fusionopolis Way, Singapore 138632 \\ \{mhdong, hli\}@i2r.a-star.edu.sg
}

\begin{abstract}
We usually build a prosody model to predict the prosodic parameters, which will be used as part of the criteria for unit selection. Spectral appropriateness of units is usually ensured by using identities of context units, which are linguistic symbols. With looking into the spectral properties of the actual signal, the spectral mismatches are often perceived in the synthetic speech. In this paper, we propose to use MFCC as spectral parameters in addition to the prosodic parameters. By introducing the spectral parameters into the criteria for unit selection, the appropriateness of units can determined by statistical models. Thus the possibility of abnormal spectral mismatches between the concatenated units can be reduced. Experiments show that the approach helps to improve the quality of synthetic speech.
\end{abstract}

Index Terms - Speech synthesis, unit selection, spectral and prosodic parameters, parameter prediction

\section{INTRODUCTION}

Unit selection approach [1][2][3][4] to speech synthesis has been shown to be one of the best approaches currently. In a unit selection based speech synthesis system, there is a large unit database which consists of many instances of the same unit as candidate units. The units in the database are designed to cover the variations of the unit as much as possible. During synthesis, a proper unit will be selected from all the candidates of the target unit. Finally the selected units are concatenated to form a speech utterance. To maintain the naturalness of the synthetic speech, prosody needs to be predicted for each unit. The prosody model predicts the prosodic parameters, which normally describe the pitch, duration and energy of speech units. These prosodic parameters are used as part of the criteria for unit selection.

However, the use of prosody alone is not enough. The prosodic parameters can only ensure the prosody appropriateness of speech. Another important aspect of natural speech, the spectral appropriateness of speech unit, is also need to be taken care of. Although currently many systems do not explicitly use spectral measures to select units, the spectrum is somewhat controlled during unit selection. The spectral appropriateness is ensured in two ways (1) use context information of the unit to make sure the selected unit is from a context similar to that of the target unit. The underlying assumption is that the units from the same context may have the similar spectral property; (2) use spectral information as part of the join cost during concatenation of units. This is done by comparing the spectrum mismatch on the boundaries of the unit that will be concatenated. However, these methods cannot effectively maintain the smoothness of speech. Acute spectral mismatches are still often perceived in the synthetic speech.

To overcome this problem, in this paper, we propose to introduce MFCC coefficients as spectral parameters into the unit selection based speech synthesis. By using MFCC coefficients, the target spectrum of units can be predicted using statistical models and be applied in unit selection process. The spectral information is normally used in statistical parametric synthesizers [5][6]. In these systems, the spectral parameters are predicted with HMM models or CART approaches. The predicted parameters are then used as parameters to synthesize speech with parametric approaches. In unit selection based speech synthesis, there are some attempts [7][8] to use spectral information to help selecting the proper units. However, the spectral information and prosodic information are separately modeled and applied in unit selection. It is difficult to balance them in the cost function.

In our approach, we include both prosodic parameters and spectral parameters into our unit selection criteria, and use statistical model to predict the parameters. The set of parameters will be used to calculate the target cost. Different from [7][8], we will integrate spectral information and prosodic information as a single acoustic vector and process them in a unified manner.

In the following sections, we will first introduce the procedures for corpus processing. Then we describe the definition of the acoustic parameters, and the method to apply them in the unit selection process. Finally, we present some experiment results.

\section{SPEECH CORPUS}


The speech corpus that we used in this research is the British English corpus that is provided by University of Edinburgh for the Blizzard Challenge 2008. The released part of the corpus consists of 15 hours speech in 9,509 utterances, which cover children stories, isolated words, emphasis carrying sentences, news articles, etc[9][10]. The corpus was designed to cover the variations of diphone as much as possible.

\subsection{Unit Labeling}

The corpus comes with transcriptions, which are contained in files of festival utterance format. The RP phone set [11] is used to define the pronunciations of the utterances. There are 50 different phonemes in the corpus.

To build the unit database for the unit selection based synthesis, we first need to extract the unit, ie, identifying the start and end points of each unit in the speech utterances. In this work, the labeling of the phone-sized unit is done with HTK automatically. A 39 dimensional MFCC feature (12 MFCC and log energy, plus delta and delta-delta features) is used for the training of the phone models. The frame size is 0.025 second and the frame shift is 0.01 second. Three states are defined for each context independent HMM model for each phone. The phone models are first trained with the speech corpus. Unit boundaries are then obtained by force alignment of speech with its phonetic sequence. The MFCC features that are used for the alignment will further be used in the later stage of the unit selected based synthesis process.

\subsection{F0 Calculation}

F0 feature is one of the most important features of prosody of speech. In this work, F0 of speech utterance is calculated with the Praat software [12]. Same as MFCC, we use a frame size of 0.01 second. The F0 values of every 0.01 second interval are calculated. For unvoiced part, interpolation is done to give a none-zero F0 value for each interval. Then we apply a simple smoothing process to this F0 sequence. The smoothing is done with moving average represented with the following formula:

$$
p_{i}^{\prime}=\left(p_{i-1}+p_{i}+p_{i+1}\right) / 3
$$

where $p_{i}$ is the $\mathrm{F} 0$ value of the $i$-th frame.

\section{METHOD}

\subsection{The Acoustic Parameters}

The acoustic parameters we define here are used as criteria for selecting the best units. Normally, the parameters are prosodic parameters that describe pitch and duration of unit. However, the use of prosody alone does not take into account the spectral mismatches. To better describe the unit for unit selection, we include both spectral parameters and prosodic parameters in our models. In this work, we use MFCC as our parameters to represent spectral information.

We use phone as the basic synthesis unit. The speech signal of each unit is separated into 3 segments, each corresponding to one of $3 \mathrm{HMM}$ states in forced alignment. We use mean values of MFCC vectors for each speech segment to represent the spectral information of the HMM state. For prosodic parameters, we only consider mean value of F0 and duration for each HMM state. Therefore, the 39 MFCC coefficients, the F0 and duration values together form a 41-dimensional vector for each state. For each unit, there are three vectors to represent the three states.

The acoustic parameters for each unit can be represented as the following:

$$
X=\left(X_{1}, X_{2}, X_{3}\right)
$$

where $X_{i}$ is a 41-dimensional vector for state $\mathrm{i}(\mathrm{i}=1,2,3)$.

\subsection{Linguistic Features}

The linguistic features are derived from input text. They are used for predicting the acoustic parameters. In this work, the corpus comes with the utterance structure for each speech file. We define the features following those that are used in HTS system [13]. We derived the following linguistic features from the utterance files (The number of parameters in each category is listed in the brackets):

- Context units: phone identities of the previous 2 and next 2 units. (4)

- Syllable information: Stress, accent, length of the previous, current and next syllables. (9)

- Syllable position information: syllable position in word and phrase, stressed syllable position in phrase, accented syllable position in phrase, distance from the stressed syllable, distance from the accented syllable, and name of the vowel in the syllable. (13)

- Word information: length and part-of-speech of the previous word, current word and next word, position of the word in phrase. (12).

- Phrase information: Lengths (in number of words and syllables) of previous phrase, current phrase and next phrase, position of the current phrase in major phrase, boundary tone of the current phase. (8)

- Utterance information: Lengths in number of syllables, words and phrases. (3)

Putting all the features together, we form an input linguistic feature vector of 53 elements.

\subsection{Acoustic Parameter Prediction}

The acoustic parameter prediction process calculates the parameters from the linguistic features. The prediction can be represented with the following formula:

$$
y_{i}=F_{i}(L)
$$


where $y_{i}$ is the $\mathrm{i}$-th parameter for the unit, $\mathrm{L}$ is the linguistic feature vector for the unit.

In this work, with the 53 linguistic features being the predictors and the $41 \times 3$ parameters ( 3 vectors for 3 states) being the responses, we build our models using CART [14] approach. Each parameter is predicted separately with a CART tree. This is different from the prediction of the vector in [7], where CART is used to predict a vector. We try to use individual trees to predict a more accurate value for each individual parameter. We understand individually predicting the parameters overlooked the correlation between the parameters. However, the parameters are automatically constrained to each other in the target cost.

\subsection{Cost Function}

The unit selection process is based on the cost function that consists of two parts (1) a target cost to measure the difference between the target unit and the candidate unit. (2) a join cost to measure the acoustic smoothness between the concatenated units.

Our target cost further consists of two parts (1) the cost of acoustic parameters and (2) the cost of context linguistic features. The target cost $c_{t}$ is defined as the following:

$$
c_{t}=w_{t a} c_{t a}+w_{t l} c_{t l}
$$

where, $c_{t a}$ and $c_{t l}$ are cost of acoustic parameters and cost of linguistic features, $w_{t a}$ and $w_{t l}$ are the weights respectively.

The reason why we use two cost components here is that each one of them alone is not sufficient to describe the target cost. The cost of linguistic feature is to ensure the general spectral and prosodic correctness of the candidate unit. However, due to the variations of speech, using this cost alone may easily leads to extreme cases (abnormal spectrum). The use of cost for acoustic parameter can avoid the selection of the extreme cases, because the statistical models favor the average values. However, the use of acoustic parameter alone is also not enough because the accuracy of prediction model is always limited.

The cost of acoustic parameters $c_{t a}$ is defined as the squared value of Mahalanobis distance [15] (i.e we did not take the square root in the following formula) between the target unit and the candidate unit is as the following:

$$
c_{t a}=\sum_{i=1}^{3}\left(\left(U_{i}-V_{i}\right)^{T} W^{-1}\left(U_{i}-V_{i}\right)\right)
$$

where $W$ is the covariance matrix, $U_{i}$ and $V_{i}(\mathrm{i}=1,2,3)$ are predicted parameter vectors for target unit and the actual parameter vector for candidate unit. In our work, $\mathrm{W}$ is a global covariance matrix that is shared by different phones. The reason why we use Mahalanobis distance is that it takes into account the correlations between the each element in the vector.

The cost of context linguistic features $c_{t l}$ is defined according to the difference between the features of the target unit and those of the candidate units. When the feature is different, a cost value is given. The total cost is the sum of all the costs for each individual features. In this function, we give higher cost value to the mismatch of important factors (e.g. the identities of immediate previous unit and immediate next unit, the accent of the unit, the stress of the unit, etc).

Join cost $c_{j}$ measures the mismatch between two units that will be concatenated. It is defined as the squared value of Mahalanobis distance between the vector of the end frame of the previous unit $E_{i-1}$ vector of the start frame of the current unit $S_{i}$

$$
c_{j}=\left(E_{i-1}-S_{i}\right)^{T} W^{-1}\left(E_{i-1}-S_{i}\right)
$$

where $W$ is the covariance matrix.

The total cost $\mathrm{c}$ is calculated with the following function.

$$
c=w_{t} \sum_{i=0}^{n} c_{t}(i)+w_{j} \sum_{i=1}^{n} c_{j}(i)
$$

where $n$ is number of units in the sequence, $\operatorname{ct}(i)$ is the target cost of unit i, cj(i) is the join cost between unit i-1 and unit $\mathrm{i}, w_{t}$ and $w_{j}$ are weights for target cost and join cost respectively.

The best unit sequence is determined by searching for a best path among the candidate unit lattice to minimize the total cost of the selected sequence. Viterbi algorithm is used to find the best sequence.

\section{EXPERIMENTS}

The corpus consists of 9,509 utterances. To reduce the computation time, in our experiment we use part of them for our training. We randomly selected 2,000 utterances from different categories of the utterances. Among the units contained in the utterances, we randomly selected 35,200 units as our training data. For each unit, the $41 \times 3$ (3 vectors for 3 states, each vector is 41 dimensional) acoustic parameters and 53 linguistic features are then calculated.

Regression tree is used to model the parameters. Each acoustic parameter is predicted separately with an individual tree. We use CART functions in Matlab to train our prediction models. For each parameter, a tree is first trained with the training data. Then it is tested with 10 -fold crossvalidation method on the training data to find the best subtree. Finally, the tree is pruned to the best sub-tree. In total, 123 trees are trained for the 3 vectors of a unit.

The weights in the cost function are manually tuned. To tune the weight to the system's best performance, we 
selected 20 testing sentences from different category. Whenever there is a new setting for the weights, we synthesize the testing sentences to see whether the speech quality is improved.

To test how the MFCC features help to improve the speech quality (compared with using pitch and duration parameters alone), we conducted a listening test on 40 randomly selected testing sentences. We compare the quality of synthesized speech based on two different definitions of the acoustic parameters. One includes MFCC, and the other does not. The two definitions are:

- Method A: We use 39 MFCC coefficients, F0 and duration for each phone state. Three 41-dimensional vectors are used to represent the three states.

- Method B: We do not use MFCC coefficients in the acoustic parameter. Instead, we use only $\mathrm{F} 0$ and duration for each phone state. Three 2-dimensional vectors are used to represent the three states.

We tuned the systems based on the two methods to their best performance as possible as we can. Then we synthesized 40 utterances with the 2 different methods for target cost calculation. Then we conduct a listening test involving 5 listeners. In the test, we ask each listener to tell which utterance in the pair is better. The result is as shown in Table 1 . From the table, we see that $72 \%$ of votes among the 200 feedbacks prefer the utterances that are synthesized based on method A. Therefore, it shows that the use of MFCC as part of criteria for unit selection helps to improve the speech quality.

Table 1. Result of Listening Test.

\begin{tabular}{|c|c|c|}
\hline Method & A & B \\
\hline $\begin{array}{c}\text { Listener's } \\
\text { preference }\end{array}$ & $72 \%$ & $28 \%$ \\
\hline
\end{tabular}

\section{CONCLUSIONS}

We introduced the acoustic parameters that include MFCC coefficients as spectral parameters in addition to the prosodic parameters for unit selection based speech synthesis. We used regression tree approach to predict the acoustic parameters for sub-unit segments at HMM state level. The cost function was defined to use the cost of acoustic parameters as one of the components. The cost for acoustic parameters is defined as the Mahalanobis distance between the respective vectors. Compared with the use of prosodic parameters alone as criteria in unit selection, the introduction of spectral parameters helps to improve the speech quality.
[1] A. W. Black, P. Taylor, "Automatically Clustering Similar Units for Unit Selection in Speech Synthesis," in Proc. Eurospeech 97, vol 2 pp 601-604, Thodes, Greece.

[2] R. Clark, K. Richmond, V. Strom, S. King, "Multisyn voice for the Blizzard Challenge 2006," Blizzard Workshop 2006.

[3] M. Schroder, A. Hunecke, S. Krstulovic, "OpenMary - Open Source Unit Selction as the Basic for Research on Expressive Synthesis," Blizzard Workshop 2006.

[4] M. Chu, H. Peng, Y. Zhao, Z. Niu and E. Chang, "Microsoft Mulan - a Bilingual TTS System”, Proc. of ICASSP 2003, Hong Kong, 2003.

[5] K. Tokuda, T. Yoshimura, T. Masuko, T. Kobayashi, and Kitamura T., "Speech Parameter Generation Algorithms for HMM-based Speech Synthesis," in ICASSP2000, Istanbul, Turkey, 2000.

[6] Alan W Black, "CLUSTERGEN: A Statistical Parametric Synthesizer using Trajectory Modeling," in Proc. Interspeech, Pittsburgh, 2006.

[7] A. W. Black, C. L. Bennett, B. C. Blanchard, J. Kominek, B. Langner, K. Prahallad and A. Toth, "CMU Blizzard 2007, A hybrid Acoustic Unit Selection System from Statistically Predicted Parameters," Blizzard Challenge workshop 2007.

[8] Zhenghua Ling et al, "The USTC and iflytek Speech Synthesis Systems for Blizzard Challenge 2007," Blizzard Challenge Workshop 2007.

[9] V. Strom, R. Clark, and S. King, "Expressive Prosody for Unit-Selection Speech Synthesis," in Proc. Interspeech, Pittsburgh, 2006.

[10] V. Strom, A. Nenkova, R. Clark, Y. Vazquez-Alvarez, J. Brenier, S. King, D. Jurafsky, "Modelling Prominence and Emphasis Improves Unit-Selection Synthesis," in Proc. Interspeech, Antwerp, 2007.

[11] S. Fitt, "Documentation and User Guide to UNISYN Lexicon and Post-lexical Rules, Tech. Rep.", Centre for Speech Technology Research, Edinburgh, 2000.

[12] Boersma, Paul, "Praat, a System for Doing Phonetics by Computer." Glot International 5:9/10, 341-345, 2001.

[13] K. Tokuda, H. Zen, A.W. Black, An HMM-based speech synthesis system applied to English, Proc. of 2002 IEEE SSW, Sept. 2002.

[14] L. Breiman, , J. H. Friedman, R. A. Olshen, and C. J. Stone, "Classification and Regression Trees". Monterey, Calif., U.S.A.: Wadsworth, Inc., 1984.

[15] P.C. Mahalanobis, On the Generalized Distance in Statistics, Proceedings of the National Institute of Science of India 12 (1936) 49-55.

\section{REFERENCES}

